\title{
Blunt traumatic rupture of the thoracic oesophagus
}

\author{
C P YOUNG, S R LARGE, S J EDMONDSON
}

From the Department of Cardiothoracic Surgery, St Bartholomew's Hospital, London

\begin{abstract}
A man with a crush injury of his upper abdomen developed bilateral pulmonary empyema after repair of tears of the oesophagus and liver. Attempts to withdraw chest drains led to recurrent septicaemia, treated by reinsertion of the drains plus administration of antibiotics. The communication of the empyema space with both the bronchial tree and the oesophagus was managed successfully with intermittent positive pressure ventilation and with a double lumen endobronchial tube isolating the right lung for 10 days. Traumatic rupture of the thoracic oesophagus carries a high mortality and prompt repair is vital.
\end{abstract}

Reports of oesophageal rupture following blunt thoracic or abdominal trauma are rare. The first, in 1936, ' described the unsuccessful result in a case of blunt traumatic rupture of the lower third of the thoracic oesophagus. A review of published reports of this unusual and therefore unsuspected injury follows the presentation of our case.

\section{Case report}

A 19 year old labourer was transferred to St Bartholomew's Hospital, having sustained a crush injury of his upper abdomen after being pinned to a wall by a dumper truck for seven minutes. His presenting complaint was of lower chest and upper abdominal pain, and on examination he had bruising over this region. His blood pressure was 90/ $60 \mathrm{~mm} \mathrm{Hg}$ and pulse rate 145 beats/minute, and he was afebrile. The chest radiograph confirmed the clinical signs of a left pleural effusion and showed no evidence of traumatic gastric herniation. A left sided intrathoracic chest drain was inserted and partially digested food was drained.

The patient was taken to the operating theatre with a presumptive diagnosis of a ruptured oesophagus. Through a left thoracolaparotomy the left pleural cavity, soiled by gastric contents, was washed out. The mediastinal pleura and disrupted lower third of the oesophagus could then be seen. The oesophageal tear was primarily repaired with two layers of prolene sutures. Bleeding was noted from below the diaphragm. This was from a linear tear in the diaphragmatic surface of the right lobe of the liver, and was repaired by direct suture via a right thoracotomy. The right chest cavity was also soiled, and was therefore cleaned and drained. His

Address for reprint requests: Mr S J Edmondson, Cardiothoracic Surgery Unit, St Bartholomew's Hospital, London EC1A 7BE.

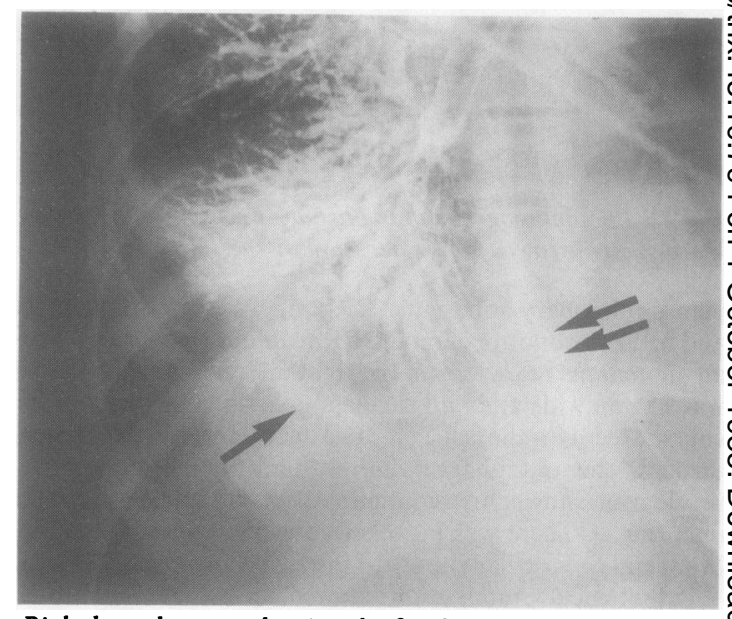

Right bronchogram showing the fistulous connection with the oesophagus and the empyema.

postoperative course was complicated. He developed bilateral empyema, which was managed by the insertion of $\vec{\partial}$ basal chest drains. Attempts to withdraw the drains gradually resulted in recurrent episodes of septicaemia. Three of these episodes led to cardiorespiratory arrests, from which he was resuscitated successfully. Each time the septicaemia resolved after reinsertion of the chest drains and the institu- $-\underset{ }{-}$ tion of broad spectrum antibiotic treatment. One period of $\frac{\sigma}{3}$ inadequate drainage of the right sided empyema and oesophageal leak led to a spontaneous discharge of the pus via the right bronchial tree, The figure shows a right bronchogram with passage of dye from the bronchial tree into the empyema 을 (single arrow). The empyema space also communicates with the oesophagus (double arrow). This was managed successfully by intermittent positive pressure ventilation with a double lumen endobronchial tube isolating the right lung for $\mathrm{N}$ 10 days. He was fed during this time through a fashioned $\mathrm{N}$ jejunostomy with supplementary intravenous feeding. Serial $\mathrm{N}$ barium swallows showed preferential flow into the stomach $\omega$ after his 86th hospital day and oral feeding was begun. By the 135 th day no oesophageal leak was apparent; shortly afterco this he was discharged home gaining weight. Subsequent $\frac{\bar{D}}{\Phi}$ review disclosed no symptoms or radiographic evidence of $\stackrel{\oplus}{?}$ oesophageal stricture formation.

\section{Discussion}

Oesophageal rupture is not uncommon, often following $\stackrel{\text { Q }}{2}$ direct trauma (iatrogenic, associated with a foreign body). It may complicate malignant tumours of the bronchus or $\delta$ 
oesophagus, or repeated vomiting. Blunt trauma is a rare cause, being found in only two of 85 cases of oesophageal perforation collected by Michel et al in 1980 and in three of 126 cases reported by Trigiani and Belsey in 1977. Since Vinson's first report in $1936^{\prime}$ several publications have described the more common rupture of the upper third of the thoracic oesophagus. ${ }^{124-7}$ With such an injury the patients present late (up to 11 months after the initial injury ${ }^{4}$ ), and they usually have signs of a tracheo-oesophageal fistula. The mortality associated with this injury was $20 \%$ in a series of 15 collected by Worman et al in 1962.' Lower oesophageal rupture caused by blunt trauma, as in the present case, is even more unusual and is associated with a very high mortality, $80 \%$. Traumatic disruption of the oesophagus is reported only in males and affects a young age group, which seems to be more at risk from high velocity trauma. Rib fractures are an uncommon association and it is postulated that people of this age group have a compliant chest wall; older people tend to sustain rib fractures and so have a different pattern of injury.

Four mechanisms of injury are proposed. Anteroposterior compression of the chest may lead to crushing of the upper oesophagus, causing a tracheo-oesophageal fistula. ${ }^{6}$ Secondly, a deceleration force can lead to disruption of some of the segmental oesophageal blood supply and ischaemic necrosis therefore follows. ${ }^{6}$ Thirdly, an abdominal compressive force may raise intraluminal oesophageal pressure; if the glottis remains closed rupture of the distal oesophagus may occur. Finally, blast injuries may also cause oesophageal rupture. The mechanism is uncertain in this unusual injury; it is, however, postulated that air under pressure forces the cricopharyngeal sphincter to give way, leading to rapid dilatation of the oesophagus. If the oesophagogastric sphincter remains closed the high pressure may cause oesophageal rupture, often in the lower third.

Associated major injuries requiring urgent action often complicate blunt thoracic trauma, allowing the oesophageal rupture to be overlooked. ${ }^{56}$ As with any other form of rupture of the thoracic oesophagus, the interval between injury and operative intervention is probably the single most important prognostic factor for the patient. The results of surgical treatment 12-18 hours after rupture are associated with a much higher leak rate from the repair or anastomosis. This is in part related to the prolonged contact with noxious gastric contents. It has also been reported that the amylase content of the pleural fluid rises substantially after 24 hours, contributing to the increased mortality. The most important factor appears to be the development of infective mediastinitis secondary to the initial chemical mediastinitis. Early recognition is therefore extremely important in this unusual condition.

\section{References}

1 Vinson PP. External trauma as a cause of lesions of the oesophagus. Am J Digest Dis 1936;3:457-9.

2 Michel L, Grillo HC, Malt RA. Operative and non-operative management of oesophageal perforations. Ann Surg 1981; 194:57-63.

3 Trigiani E, Belsey R. Oesophageal trauma: incidence, diagnosis and management. Thorax 1977;32:241-9.

4 Gupta RL, Bannerjee T. Traumatic oesophagotracheal fistula. Am J Surg 1961;101:227-9.

5 Worman LW, Hurley JD, Pemberton AH, et al. Rupture of the oesophagus from external blunt trauma. Arch Surg 1962; 85:333-8.

6 Chilimindris CP. Rupture of the thoracic oesophagus from blunt trauma. J Trauma 1977;17:968-71.

7 Stothert JC, Buttorff J, Kaminski DL. Thoracic oesophageal and tracheal injury following blunt trauma. J Trauma 1980;20: 992-5. 